\title{
Article \\ Optimal Control Problems Involving Combined Fractional Operators with General Analytic Kernels ${ }^{+}$
}

\author{
Faïçal Ndaïrou $\ddagger$ (D) and Delfim F. M. Torres *, $\ddagger$
}

Citation: Ndaïrou, F.; Torres, D.F.M. Optimal Control Problems Involving Combined Fractional Operators with General Analytic Kernels. Mathematics 2021, 9, 2355. https://doi.org/10.3390/ math9192355

Academic Editors: William S. Oates and Somayeh Mashayekhi

Received: 5 September 2021

Accepted: 19 September 2021

Published: 22 September 2021

Publisher's Note: MDPI stays neutral with regard to jurisdictional claims in published maps and institutional affiliations.

Copyright: (c) 2021 by the authors. Licensee MDPI, Basel, Switzerland. This article is an open access article distributed under the terms and conditions of the Creative Commons Attribution (CC BY) license (https:// creativecommons.org/licenses/by/ $4.0 /)$.
Center for Research and Development in Mathematics and Applications (CIDMA), Department of Mathematics, University of Aveiro, 3810-193 Aveiro, Portugal; faical@ua.pt

* Correspondence: delfim@ua.pt; Tel.: +351-234-370-668

† This research is part of first author's Ph.D. project, which is carried out at the University of Aveiro under the Doctoral Program in Applied Mathematics of Universities of Minho, Aveiro, and Porto (MAP-PDMA).

$\ddagger$ These authors contributed equally to this work.

\begin{abstract}
Fractional optimal control problems via a wide class of fractional operators with a general analytic kernel are introduced. Necessary optimality conditions of Pontryagin type for the considered problem are obtained after proving a Gronwall type inequality as well as results on continuity and differentiability of perturbed trajectories. Moreover, a Mangasarian type sufficient global optimality condition for the general analytic kernel fractional optimal control problem is proved. An illustrative example is discussed.
\end{abstract}

Keywords: fractional operators with general analytic kernels; Gronwall's inequality; optimal control and Pontryagin's extremals; Mangasarian sufficient optimality condition

MSC: 26A33; 49K15

\section{Introduction}

Fractional Calculus, as a generalization of the traditional calculus through derivation and integration of an arbitrary order, is a rapidly growing field of mathematical research. Indeed, due to the existence of many different fractional operators in the literature, there is an interest in defining a more general class of fractional operators, which include existing operators as particular cases. This is important in the sense that with a general framework of operators it might be possible to establish a mathematical theory for this general formalism, rather than considering specific models with particular results. In this direction, Fernandez, Özarslan and Baleanu proposed in 2019 a fractional integral operator, based on a general analytic kernel, that includes a number of existing and known operators [1]. Since this seminal work of 2019, several interesting results appeared, e.g., determination of source terms for fractional Rayleigh-Stokes equations with random data [2], new analytic properties of tempered fractional calculus [3], simulation of nonlinear dynamics with fractional neural networks arising in the modeling of cognitive decision making processes [4], new numerical methods for variable order fractional nonlinear quadratic integro-differential equations [5], and analysis of impulsive $\varphi$-Hilfer fractional differential equations [6]. Here, we investigate, for the first time in the literature, optimal control problems that involve a combined Caputo fractional derivative with a general analytic kernel in the sense of Fernandez, Özarslan and Baleanu.

The subject of combined fractional derivatives deals with the issue of combining the past and the future of the modelling process into one single operator. This is done by a convex combination of the left and right fractional derivatives. The idea was firstly introduced in [7] by Malinowska and Torres, following a previous idea of Klimek [8], and then further investigated by the authors in [9-11]. See also [12-14] and references therein. As mentioned in [9], one advantage of combining fractional derivatives lie in the fact that 
they allow to describe a more general class of variational problems. Thus, it seems natural to consider optimal control problems that involve combined fractional derivatives with a general analytic kernel.

It should be mentioned that there is a rich literature on optimal control with fractional operators. Recent results include, for example: (i) sensitivity properties of optimal control problems governed by nonlinear Hilfer fractional evolution inclusions in Hilbert spaces [15]; (ii) existence of a solution for a class of fractional delayed stochastic differential equations with non-instantaneous impulses and fractional Brownian motions [16]; (iii) an optimal control analysis of a fractional COVID-19 epidemic model to minimize the infection and maximize susceptible individuals under the Atangana-Baleanu fractional operator in the Caputo sense [17]; (iv) a Pontryagin maximum principle for optimal control problems with concentrated parameters for a degenerate differential equation with the Caputo operator [18]; etc. However, no available results on optimal control, with combined general analytic kernels, exist in the literature. For non-combined general analytic kernels, optimal control results are scarce and restricted to the recent publication [19]. There, a weak version of Pontryagin's Maximum Principle for optimal control problems involving a general analytic kernel is given but the emphasis is on the classical setting of the calculus of variations (e.g., isoperimetric variational problems) and with results valid only in the class of piece-wise continuous differentiable state trajectories and piece-wise continuous controls [19]. In contrast, our current results are more general, being valid in the class of absolutely continuous state trajectories and $L^{2}$ controls. Moreover, results of [19] are valid only in the absence of constraints on the values of the controls; that is, the controls take values in all the Euclidean space while here we are able to deal with more general and challenge situations when the controls may take values in any time-dependent close convex set of $L^{2}$.

The manuscript is organized as follows. Section 2 presents preliminary notions and results needed in the sequel, and follows the original results of the paper (Sections 3 and 4). In Section 3, we prove two results that are fundamental in the development of our work: a duality relation (Lemma 5) and integration by parts formulas (Lemma 7). The main results appear then in Section 4, where we state and prove a Gronwall type inequality (Theorem 1) and, as application to this inequality, we prove two results: a result on the continuity of solutions (see Lemma 8 and Corollary 1 ) and a necessary optimality condition of Pontryagin type to an optimal control problem with a general analytic kernel in the sense of Fernandez, Özarslan and Baleanu (Theorem 2). We end Section 4 by proving a sufficient condition for global optimality (Theorem 3). An example, illustrating the applicability of the obtained results, is given (see Examples 1 and 2). Finally, Section 5 give the main conclusions of the paper, including some possible future directions of research.

\section{Preliminaries}

In this section, we recall the definitions of fractional operators based on general analytic kernels and state some of their properties, relevant to our work.

Definition 1 (See [1]). Let $[a, b]$ be a real interval, $\alpha$ be a real parameter in $[0,1], \beta$ be a complex parameter with non-negative real part, and $R$ be a positive number satisfying $R>(b-a)^{\operatorname{Re}(\beta)}$. Let $A$ be a complex function, analytic on the disc $D(0, R)$, and defined on this disc by the locally uniformly convergent power series

$$
A(x)=\sum_{n=0}^{\infty} a_{n} x^{n}
$$

The left and right-sided fractional integrals with general analytic kernels of a locally integrable function $x:[a, b] \rightarrow \mathbb{R}$ (that is, $x \in L^{1}([a, b], \mathbb{R})$ ) are defined by

$$
{ }^{A} I_{a+}^{\alpha, \beta} x(t):=\int_{a}^{t}(t-s)^{\alpha-1} A\left((t-s)^{\beta}\right) x(s) d s
$$


and

$$
{ }^{A} I_{b-}^{\alpha, \beta} x(t):=\int_{t}^{b}(s-t)^{\alpha-1} A\left((s-t)^{\beta}\right) x(s) d s,
$$

respectively.

Notation 1. For any analytic function $A$ as in Definition 1 , we define $A_{\Gamma}$ as

$$
A_{\Gamma}(x):=\sum_{n=0}^{\infty} a_{n} \Gamma(\beta n+\alpha) x^{n} .
$$

Lemma 1 (Series formula [1]). For any integrable function $x \in L^{1}([a, b], \mathbb{R})$, the following uniformly convergent series formulas for ${ }^{A} I_{a+}^{\alpha, \beta} x$ and for ${ }^{A} I_{b-}^{\alpha, \beta} x$ as functions on $[a, b]$ hold:

$$
{ }^{A} I_{a+}^{\alpha, \beta} x(t):=\sum_{n=0}^{\infty} a_{n} \Gamma(\beta n+\alpha)^{R L} I_{a+}^{\alpha+n \beta} x(t)
$$

and

$$
{ }^{A} I_{b-}^{\alpha, \beta} x(t):=\sum_{n=0}^{\infty} a_{n} \Gamma(\beta n+\alpha)^{R L} I_{b-}^{\alpha+n \beta} x(t),
$$

where ${ }^{A} I_{a+}^{\alpha+n \beta}$ and ${ }^{A} I_{b-}^{\alpha+n \beta}$ are, respectively, the left and right-sided Riemann-Liouville fractional integrals of order $\alpha+n \beta$.

Lemma 2 (Theorem 2.5 of [1]). With all notations as in Definition 1, we have a well bounded operator

$$
{ }^{A} I_{a+}^{\alpha, \beta}: L^{1}([a, b], \mathbb{R}) \rightarrow L^{1}([a, b], \mathbb{R})
$$

for any fixed $\alpha$ and $\beta$ with $\operatorname{Re}(\alpha), \operatorname{Re}(\beta) \geqslant 0$. Moreover, the operator norm denoted by $\|\cdot\|$ is obtained as

$$
\left\|{ }^{A} I_{a+}^{\alpha, \beta}\right\|=\sup _{f \in L^{1}([a, b], \mathbb{R})} \frac{\left\|{ }^{A} I_{a+}^{\alpha, \beta} f\right\|_{1}}{\|f\|_{1}}=(b-a)^{\alpha} M, \quad M=\sup _{|x|<(b-a)^{\alpha}} A(x) .
$$

In addition, similar results hold for the right-sided operator given in Definition 1, that is, the operator ${ }^{A} I_{b-}^{\alpha, \beta}$ is bounded on $L^{1}([a, b], \mathbb{R})$ with operator norm at most $(b-a)^{\alpha} M$.

Lemma 3 (Semi group property [1]). Let $a, b, A$ be as in Definition 1 , and fix $\alpha_{1}, \alpha_{2}, \beta \in \mathbb{C}$ with non-negative real parts. The semigroup property

$$
{ }^{A} I_{a+}^{\alpha_{1}, \beta} \circ{ }^{A} I_{a+}^{\alpha_{2}, \beta} x(t)={ }^{A} I_{a+}^{\alpha_{1}+\alpha_{2}, \beta} x(t)={ }^{A} I_{a+}^{\alpha_{2}+\alpha_{1}, \beta} x(t)={ }^{A} I_{a+}^{\alpha_{2}, \beta} \circ{ }^{A} I_{a+}^{\alpha_{1}, \beta} x(t)
$$

is uniformly valid (regardless of $\alpha_{1}, \alpha_{2}, \beta$ and $x$ ) if and only if the condition

$$
\sum_{m+n=k} a_{n}\left(\alpha_{1}, \beta\right) a_{m}\left(\alpha_{2}, \beta\right) \Gamma\left(\alpha_{1}+n \beta\right) \Gamma\left(\alpha_{2}+n \beta\right)=a_{k}\left(\alpha_{1}+\alpha_{2}, \beta\right) \Gamma\left(\alpha_{1}+\alpha_{2}+k \beta\right)
$$

is satisfied for all non-negative integers $k$.

A similar result to that of Lemma 3 holds for the right-sided Riemann-Liouville fractional integral operator given in Definition 1.

Next, we give some recalls on fractional derivatives with general analytic kernel in the sense of Riemann-Liouville and Caputo.

Let $a, b, \alpha, \beta$ and $A$ be as in Definition 1 and denote

$$
L^{\alpha, \beta}([a, b], \mathbb{R}):=\left\{x \in L^{1}([a, b], \mathbb{R}):{ }^{\bar{A}} I_{a+}^{1-\alpha, \beta} x,{ }^{\bar{A}} I_{b-}^{1-\alpha, \beta} x \in A C([a, b], \mathbb{R})\right\},
$$


where $A C([a, b], \mathbb{R})$ represents the set of absolutely continuous functions on $[a, b]$.

Definition 2 (See [1]). The left and right Rieman-Liouville fractional derivatives with general analytic kernels, of a function $x \in L^{\alpha, \beta}([a, b], \mathbb{R})$, are defined by

$$
{ }_{R L}^{A} D_{a+}^{\alpha, \beta} x(t)=\frac{d}{d t}\left({ }^{\bar{A}} I_{a+}^{1-\alpha, \beta} x(t)\right) \quad \text { and } \quad{ }_{R L}^{A} D_{b-}^{\alpha, \beta} x(t)=-\frac{d}{d t}\left({ }^{\bar{A}} I_{b-}^{1-\alpha, \beta} x(t)\right),
$$

where function $\bar{A}$ used on the right-hand side is an analytic function defined by

$$
\bar{A}(x)=\sum_{n=0}^{\infty} \bar{a}_{n} x^{n}
$$

and such that $A_{\Gamma} \cdot \bar{A}_{\Gamma}=1$

Now, let us denote by $A C^{\alpha, \beta}([a, b], \mathbb{R})$ the set of absolutely continuous functions that can be represented as

$$
x(t)=x(a)+{ }^{\bar{A}} I_{a+}^{\alpha, \beta} f(t) \quad \text { and } \quad x(t)=x(b)+{ }^{\bar{A}} I_{b-}^{\alpha, \beta} f(t),
$$

for some function $f \in L^{\alpha, \beta}([a, b], \mathbb{R})$.

Definition 3. The left and right Caputo fractional derivatives with general analytic kernels, of a function $x \in A C^{\alpha, \beta}([a, b], \mathbb{R})$, are defined by

$$
{ }_{C}^{A} D_{a+}^{\alpha, \beta} x(t)=\frac{d}{d t}\left[{ }^{\bar{A}} I_{a+}^{1-\alpha, \beta}(x(t)-x(a))\right]
$$

and

$$
{ }_{C}^{A} D_{b-}^{\alpha, \beta} x(t)=-\frac{d}{d t}\left[{ }^{\bar{A}} I_{b-}^{1-\alpha, \beta}(x(t)-x(b))\right],
$$

where function $\bar{A}$ used on the right-hand side is an analytic function given by $\bar{A}(x)=\sum_{n=0}^{\infty} \bar{a}_{n} x^{n}$ and such that $A_{\Gamma} \cdot \bar{A}_{\Gamma}=1$.

We would like to emphasize that, from (1) and by using the semi group property (Lemma 3), one has

$$
{ }^{\bar{A}} I_{a+}^{1-\alpha, \beta}(x(t)-x(a))={ }^{\bar{A}} I_{a+}^{1, \beta} f(t) \quad \text { and } \quad{ }^{\bar{A}} I_{b-}^{1-\alpha, \beta}(x(t)-x(b))={ }^{\bar{A}} I_{b-}^{1, \beta} f(t) .
$$

Therefore, by Definition 3 and the fact that $f \in L^{\alpha, \beta}([a, b], \mathbb{R})$, it is obvious that ${ }_{C}^{A} D_{a+}^{\alpha, \beta}$ and ${ }_{C}^{A} D_{b-}^{\alpha, \beta}$ belong to $L^{1}([a, b], \mathbb{R})$.

Lemma 4 (See, e.g., [20,21]). Let $h: \mathbb{R}^{n} \rightarrow \mathbb{R}$ be a continuously differentiable function. Then $h$ is a concave function if and only if it satisfies the so called gradient inequality:

$$
h\left(\theta_{1}\right)-h\left(\theta_{2}\right) \geq \nabla h\left(\theta_{1}\right)\left(\theta_{1}-\theta_{2}\right)
$$

for all $\theta_{1}, \theta_{2} \in \mathbb{R}^{n}$.

\section{Fundamental Properties}

We prove rules of fractional integration by parts for the general analytic kernel operators. Firstly, we show a duality formula for the integral operator (Lemma 5). Then, we use the duality formula to prove the fractional integration by parts formulas for the general analytic kernel fractional operators (Lemma 7). 
Lemma 5 (Duality relation). Let $\alpha$ and $\beta$ be as in Definition 1 with $|\alpha+\beta| \geq 1$. For any functions $x(t)$ and $y(t), t \in[a, b]$, with $x, y \in L^{1}([a, b], \mathbb{R})$, the following duality relation holds:

$$
\int_{a}^{b} x(t)^{A} I_{a+}^{\alpha, \beta} y(t) d t=\int_{a}^{b} y(t)^{A} I_{b-}^{\alpha, \beta} x(t) d t
$$

Proof. By the series formula, we know that

$$
\int_{a}^{b} x(t)^{A} I_{a+}^{\alpha, \beta} y(t) d t=\int_{a}^{b} x(t) \sum_{n=0}^{\infty} a_{n} \Gamma(\beta n+\alpha)^{R L} I_{a+}^{\alpha+n \beta} y(t) d t
$$

Since the series in the right hand side of (2) is uniformly convergent, it follows that

$$
\int_{a}^{b} x(t)^{A} I_{a+}^{\alpha, \beta} y(t) d t=\sum_{n=0}^{\infty} a_{n} \Gamma(\beta n+\alpha) \int_{a}^{b} x(t)^{R L} I_{a+}^{\alpha+n \beta} y(t) d t
$$

and, by the well-known duality of the Riemann-Liouville integral operators, which is valid under assumption $|\alpha+\beta| \geq 1$ (cf. Lemma 2.7(a) of [22]), we have

$$
\int_{a}^{b} x(t)^{R L} I_{a+}^{\alpha+n \beta} y(t) d t=\int_{a}^{b} y(t)^{R L} I_{b-}^{\alpha+n \beta} x(t) d t
$$

for any $n \in \mathbb{N}$, which leads to

$$
\sum_{n=0}^{\infty} a_{n} \Gamma(\beta n+\alpha) \int_{a}^{b} x(t)^{R L} I_{a+}^{\alpha+n \beta} y(t) d t=\sum_{n=0}^{\infty} a_{n} \Gamma(\beta n+\alpha) \int_{a}^{b} y(t)^{R L} I_{b-}^{\alpha+n \beta} x(t) d t
$$

Therefore, we obtain that

$$
\int_{a}^{b} x(t)^{A} I_{a+\beta}^{\alpha, \beta} y(t) d t=\int_{a}^{b} y(t)^{A} I_{b-}^{\alpha, \beta} x(t) d t
$$

This concludes the proof.

Lemma 6. Let $x \in A C^{\alpha, \beta}([a, b], \mathbb{R})$. The left and right sided Caputo fractional derivatives, as defined in Definition 3, coincide with the following representation:

$$
{ }_{C}^{A} D_{a+}^{\alpha, \beta} x(t)={ }^{\bar{A}} I_{a+}^{1-\alpha, \beta} x^{\prime}(t) \quad \text { and } \quad{ }_{C}^{A} D_{b-}^{\alpha, \beta} x(t)=-\left({ }^{\bar{A}} I_{b-}^{1-\alpha, \beta} x^{\prime}(t)\right) .
$$

Proof. We have, by Definition 3, that

$$
{ }_{C}^{A} D_{a+}^{\alpha, \beta} x(t)=\frac{d}{d t}\left[\bar{A} I_{a+}^{1-\alpha, \beta}(x(t)-x(a))\right]
$$

Using the series formula (Lemma 2), it follows that

$$
\begin{aligned}
{ }_{C}^{A} D_{a+}^{\alpha, \beta} x(t) & =\frac{d}{d t}\left\{\sum_{n=0}^{\infty} \bar{a}_{n} \Gamma(\beta n+1-\alpha)^{R L} I_{a+}^{\beta n+1-\alpha}(x(t)-x(a))\right\} \\
& =\frac{d}{d t}\left\{\sum_{n=0}^{\infty} \bar{a}_{n} \Gamma(\beta n+1-\alpha)\left[\frac{1}{\Gamma(\beta n+1-\alpha)} \int_{a}^{t}(t-s)^{\beta n-\alpha}(x(s)-x(a)) d s\right]\right\} .
\end{aligned}
$$


Moreover, by the classical integration by parts formula, we have

$$
\begin{aligned}
\int_{a}^{t}(t-s)^{\beta n-\alpha}(x(s)-x(a)) d s= & {\left[-\frac{1}{\beta n+1-\alpha}(t-s)^{\beta n+1-\alpha}(x(s)-x(a))\right]_{a}^{t} } \\
& +\int_{a}^{t} \frac{1}{\beta n+1-\alpha}(t-s)^{\beta n+1-\alpha} \frac{d}{d s}(x(s)-x(a)) d s \\
= & \int_{a}^{t} \frac{1}{\beta n+1-\alpha}(t-s)^{\beta n+1-\alpha} x^{\prime}(s) d s .
\end{aligned}
$$

Therefore,

$$
\begin{aligned}
{ }_{C}^{A} D_{a+}^{\alpha, \beta} x(t)=\frac{d}{d t}\left\{\sum_{n=0}^{\infty} \bar{a}_{n} \Gamma(\beta n+1-\alpha)\right. \\
\left.\times\left[\frac{1}{\Gamma(\beta n+1-\alpha)} \int_{a}^{t} \frac{1}{\beta n+1-\alpha}(t-s)^{\beta n+1-\alpha} x^{\prime}(s) d s\right]\right\} .
\end{aligned}
$$

Since the series is uniformly convergent, we can differentiate with respect to $t$ to obtain

$$
\begin{aligned}
{ }_{C}^{A} D_{a+}^{\alpha, \beta} x(t) & =\sum_{n=0}^{\infty} \bar{a}_{n} \Gamma(\beta n+1-\alpha)\left[\frac{1}{\Gamma(\beta n+1-\alpha)} \int_{a}^{t}(t-s)^{\beta n-\alpha} x^{\prime}(s) d s\right] \\
& =\sum_{n=0}^{\infty} \bar{a}_{n} \Gamma(\beta n+1-\alpha)\left[{ }^{R L} I_{a+}^{\beta n+1-\alpha} x^{\prime}(t)\right]={ }^{\bar{A}} I_{a+}^{1-\alpha, \beta} x^{\prime}(t) .
\end{aligned}
$$

In a similar way, it is possible to derive the right sided representation.

The next result is, as we shall see, an important tool for proving necessary optimality conditions to optimal control problems.

Lemma 7 (Integration by parts formula). Let $\alpha$ and $\beta$ be as in Definition 1 with $|\alpha+\beta| \geq 1$, $x \in L^{\alpha, \beta}([a, b], \mathbb{R})$ and $y \in A C^{\alpha, \beta}([a, b], \mathbb{R})$. Then, the following two formulas hold:

$$
\int_{a}^{b} x(t)_{C}^{A} D_{a+}^{\alpha, \beta} y(t) d t=\left[y(t)^{\bar{A}} I_{b-}^{1-\alpha, \beta} x(t)\right]_{a}^{b}+\int_{a}^{b} y(t)_{R L}^{A} D_{b-}^{\alpha, \beta} x(t) d t
$$

and

$$
\int_{a}^{b} x(t)_{C}^{A} D_{b^{-}}^{\alpha, \beta} y(t) d t=\left[-y(t)^{\bar{A}} I_{a^{+}}^{1-\alpha, \beta} x(t)\right]_{a}^{b}+\int_{a}^{b} y(t)_{R L}^{A} D_{a^{+}}^{\alpha, \beta} x(t) d t .
$$

Proof. By definition,

$$
\int_{a}^{b} x(t)_{C}^{A} D_{a+}^{\alpha, \beta} y(t) d t=\int_{a}^{b} x(t)^{\bar{A}} I_{a+}^{1-\alpha, \beta} y^{\prime}(t) d t
$$

and, by the duality formula of Lemma 5 , it follows that

$$
\int_{a}^{b} x(t)^{\bar{A}} I_{a+}^{1-\alpha, \beta} y^{\prime}(t) d t=\int_{a}^{b} y^{\prime}(t)^{\bar{A}} I_{b-}^{1-\alpha, \beta} x(t) d t .
$$

Using (standard) integration by parts, we obtain that

$$
\int_{a}^{b} y^{\prime}(t)^{\bar{A}} I_{b-}^{1-\alpha, \beta} x(t) d t=\left[y(t)^{\bar{A}} I_{b-}^{1-\alpha, \beta} x(t)\right]_{a}^{b}-\int_{a}^{b} y(t) \frac{d}{d t}\left({ }^{\bar{A}} I_{b-}^{1-\alpha, \beta} x(t)\right) d t
$$

which leads to the desired formula. The proof of (5) is similar. 


\section{Main Results}

In this section, following the definition given in [7], we propose a combined fractional operator for a general analytic kernel and study the related optimal control problem.

Definition 4. Let $\gamma \in[0,1]$. The combined fractional operator with general analytic kernel is defined by

$$
{ }_{C}^{A} D_{a, b}^{\alpha, \beta, \gamma}=\gamma_{C}^{A} D_{a^{+}}^{\alpha, \beta}+(1-\gamma){ }_{C}^{A} D_{b^{-}}^{\alpha, \beta} .
$$

Note that ${ }_{C}^{A} D_{a, b}^{\alpha, \beta, 0}={ }_{C}^{A} D_{b^{-}}^{\alpha, \beta}$ and ${ }_{C}^{A} D_{a, b}^{\alpha, \beta, 1}={ }_{C}^{A} D_{a^{+}}^{\alpha, \beta}$. The operator is obviously linear. Using Lemma 7 , with $x \in L^{\alpha, \beta}([a, b], \mathbb{R})$ and $y \in A C^{\alpha, \beta}([a, b], \mathbb{R})$, we can easily establish the following integration by parts formula:

$$
\begin{aligned}
\int_{a}^{b} x(t)_{C}^{A} D_{a, b}^{\alpha, \beta, \gamma} y(t) d t= & \gamma\left[y(t)^{\bar{A}} I_{b^{-}}^{1-\alpha, \beta} x(t)\right]_{a}^{b} \\
& +(1-\gamma)\left[-y(t)^{\bar{A}} I_{a^{+}}^{1-\alpha, \beta} x(t)\right]_{a}^{b}+\int_{a}^{b} y(t)_{R L}^{A} D_{a, b}^{\alpha, \beta, 1-\gamma} x(t) d t,
\end{aligned}
$$

where ${ }_{R L}^{A} D_{a, b}^{\alpha, \beta, \gamma}=\gamma_{R L}^{A} D_{a^{+}}^{\alpha, \beta}+(1-\gamma)_{R L}^{A} D_{b^{-}}^{\alpha, \beta}$.

In the following subsection, we prove a new integral inequality of Gronwall type that will be useful to investigate continuity of solutions to our optimal control problem.

\subsection{Gronwall's Inequality}

Gronwall's inequality is an important integral inequality that is often used to prove qualitative and quantitative properties of solutions to differential equations. Very recently, there were several works devoted to this subject in the field of fractional calculus: see, e.g., $[23,24]$ and references therein. The next result is a new Gronwall type inequality for a fractional integral operator with a general analytic kernel.

Theorem 1 (Gronwall's inequality). Let $f(\cdot), u(\cdot) \in L^{1}([a, b], \mathbb{R})$ be non-negative and $g(\cdot)$ be a non-negative monotonic increasing continuous function on $[a, b]$ satisfying

$$
\max _{t \in[a, b]} g(t)<\frac{1}{(b-a)^{\alpha-1} M} \quad \text { with } \quad M=\sup _{|x|<(b-a)^{\alpha}} A(x) .
$$

If

$$
u(t) \leq f(t)+g(t)\left({ }^{A} I_{a, b}^{\alpha, \beta, \gamma} u\right)(t), \quad t \in[a, b] \text { a.e., }
$$

then for almost all $t \in[a, b]$ we have

$$
u(t) \leq f(t)+\sum_{k=1}^{\infty}[g(t)]^{k}\left[\left({ }^{A} I_{a, b}^{\alpha, \beta, \gamma}\right)^{k}(f)\right](t)
$$

where ${ }^{A} I_{a, b}^{\alpha, \beta, \gamma}=\gamma^{A} I_{a^{+}}^{\alpha, \beta}+(1-\gamma)^{A} I_{b^{-}}^{\alpha, \beta}$ with $\gamma \in[0,1]$ and we use the composition of operators' notation:

$$
\left({ }^{A} I_{a, b}^{\alpha, \beta, \gamma}\right)^{k}=o_{i=1}^{k}\left({ }^{A} I_{a, b}^{\alpha, \beta, \gamma}\right)_{i}
$$

Proof. Since the operator ${ }^{A} I_{a, b}^{\alpha, \beta, \gamma}$ is a non-decreasing operator, as linear combination of non-decreasing operators, it follows that

$$
\begin{aligned}
\left({ }^{A} I_{a, b}^{\alpha, \beta, \gamma} u\right)(t) & \leq{ }^{A} I_{a, b}^{\alpha, \beta, \gamma}\left(f(\cdot)+g(t)\left({ }^{A} I_{a, b}^{\alpha, \beta, \gamma} u\right)\right)(t) \\
& =\left({ }^{A} I_{a, b}^{\alpha, \beta, \gamma} f\right)(t)+g(t)\left[\left({ }^{A} I_{a, b}^{\alpha, \beta, \gamma}\right)^{2}(u)\right](t) .
\end{aligned}
$$


Now, we substitute this previous inequality into (7), to obtain that

$$
u(t) \leq f(t)+g(t)\left({ }^{A} I_{a, b}^{\alpha, \beta, \gamma} f\right)(t)+[g(t)]^{2}\left[\left({ }^{A} I_{a, b}^{\alpha, \beta, \gamma}\right)^{2}(u)\right](t) .
$$

Repeating this procedure up to $N$ times, we get

$$
u(t) \leq f(t)+\sum_{k=1}^{N-1}(g(t))^{k}\left[\left({ }^{A} I_{a, b}^{\alpha, \beta, \gamma}\right)^{k}(f)\right](t)+(g(t))^{N}\left[\left({ }^{A} I_{a, b}^{\alpha, \beta, \gamma}\right)^{N}(u)\right](t) .
$$

Therefore, when $N \rightarrow \infty$, one has

$$
u(t) \leq f(t)+\sum_{k=1}^{\infty}(g(t))^{k}\left[\left({ }^{A} I_{a, b}^{\alpha, \beta, \gamma}\right)^{k}(f)\right](t)+\lim _{N \rightarrow \infty}(g(t))^{N}\left[\left({ }^{A} I_{a, b}^{\alpha, \beta, \gamma}\right)^{N}(u)\right](t) .
$$

To obtain the desired result, it remains to show that the series

$$
\sum_{k=1}^{\infty}(g(t))^{k}\left[\left({ }^{A} I_{a, b}^{\alpha, \beta, \gamma}\right)^{k}(f)\right](t)
$$

converges and the limit

$$
\lim _{N \rightarrow \infty}(g(t))^{N}\left[\left({ }^{A} I_{a, b}^{\alpha, \beta, \gamma}\right)^{N}(u)\right](t)
$$

is equal to zero. Let us study the composition of operators $\left({ }^{A} I_{a, b}^{\alpha, \beta, \gamma}\right)^{k}$. For this purpose, note that, by Lemma 2, the operators ${ }^{A} I_{a^{+}}^{\alpha, \beta}$ and ${ }^{A} I_{b^{-}}^{\alpha, \beta}$ are both bounded on $L^{1}([a, b], \mathbb{R})$. Therefore, because $L^{1}([a, b], \mathbb{R})$ is a norm vector space, we have that the linear combination $\gamma^{A} I_{a^{+}}^{\alpha, \beta}+(1-\gamma)^{A} I_{b^{-}}^{\alpha, \beta}={ }^{A} I_{a, b}^{\alpha, \beta, \gamma}$ is also a bounded operator on $L^{1}([a, b], \mathbb{R})$ having the same operator norm, precisely

$$
\left\|{ }^{A} I_{a, b}^{\alpha, \beta, \gamma} f\right\|_{1} \leqslant(b-a)^{\alpha} M\|f\|_{1}, \quad f \in L^{1}([a, b], \mathbb{R}) .
$$

As a consequence, if $f \in L^{1}([a, b], \mathbb{R})$, then for any fixed integer $k$ we have that the composition $\left({ }^{A} I_{a, b}^{\alpha, \beta, \gamma}\right)^{k}(f) \in L^{1}([a, b], \mathbb{R})$ and is bounded in the sense that

$$
\left\|\left({ }^{A} I_{a, b}^{\alpha, \beta, \gamma}\right)^{k} f\right\|_{1} \leqslant\left[(b-a)^{\alpha} M\right]^{k}\|f\|_{1}, \quad f \in L^{1}([a, b], \mathbb{R}) .
$$

Moreover, using the mean value theorem, we have that there exists $t \in[a, b]$ such that

$$
\left|\left({ }^{A} I_{a, b}^{\alpha, \beta, \gamma}\right)^{k}(f)(t)\right|=\frac{1}{(b-a)}\left\|\left({ }^{A} I_{a, b}^{\alpha, \beta, \gamma}\right)^{k} f\right\|_{1} .
$$

Hence, we obtain that

$$
\left|\left({ }^{A} I_{a, b}^{\alpha, \beta, \gamma}\right)^{k}(f)(t)\right| \leqslant \frac{1}{(b-a)}\left[(b-a)^{\alpha} M\right]^{k}\|f\|_{1}, \quad f \in L^{1}([a, b], \mathbb{R}),
$$

and it follows that

$$
\left|\sum_{k=1}^{\infty}(g(t))^{k}\left({ }^{A} I_{a, b}^{\alpha, \beta, \gamma}\right)^{k}(f)(t)\right| \leq\|f\|_{1} \sum_{k=1}^{\infty}\left[(b-a)^{\alpha-1} M N\right]^{k}, \quad f \in L^{1}([a, b], \mathbb{R}),
$$


where $N=\max _{t \in[a, b]} g(t)$. Finally, the series converges since, by assumptions,

$$
N<\frac{1}{(b-a)^{\alpha-1} M}
$$

Moreover, according to the necessary condition of convergence of an infinite series, one deduces that

$$
\lim _{k \rightarrow \infty}(g(t))^{k}\left({ }^{A} I_{a, b}^{\alpha, \beta, \gamma}\right)^{k}(f)(t)=0 .
$$

This concludes the proof.

\subsection{Applications}

In this subsection, we prove several important results: continuity of solutions to optimal control problems (Lemma 8), which is an application of our Gronwall's inequality (Theorem 1); differentiability of the perturbed trajectories (Corollary 1); and a necessary optimality condition of Pontryagin type to problem (8) (Theorem 2), which happens to be an application of the results on continuity, differentiability, and integration by parts. First of all, let us define the optimal control problem that we will be studying.

We consider an analytic kernel fractional optimal control problem, which consists in finding a control $u \in \Omega(t) \subseteq L^{2}([a, b], \mathbb{R})$ and its corresponding state trajectory $x \in A C^{\alpha, \beta}$ solution to the following problem:

$$
\begin{aligned}
& J[x(\cdot), u(\cdot)]=\int_{a}^{b} L(t, x(t), u(t)) d t \longrightarrow \max , \\
& { }_{C}^{A} D_{a, b}^{\alpha, \beta, \gamma} x(t)=f(t, x(t), u(t)), \quad t \in[a, b], \quad|\alpha+\beta| \geq 1, \\
& x(\cdot) \in A C^{\alpha, \beta}, \quad u(\cdot) \in \Omega(t) \subseteq L^{2}, \quad \text { a.e. } t \in[a, b], \\
& x(a)=x_{a} \text {, }
\end{aligned}
$$

where $\Omega(t)$ is a closed convex subset of $L^{2}$ and functions $L$ and $f$ are assumed to be continuously differentiable in all their three arguments, that is, $f \in C^{1}$ and $L \in C^{1}$. In particular, $f$ is locally Lipschitz with Lipschitz constant $K$. By solution of the analytic kernel fractional optimal control problem (8), we mean a pair $(x(\cdot), u(\cdot)) \in A C^{\alpha, \beta} \times \Omega(t)$ satisfying the control system ${ }_{C}^{A} D_{a, b}^{\alpha, \beta, \gamma} x(t)=f(t, x(t), u(t)), t \in[a, b]$, the initial condition $x(a)=x_{a}$, and giving maximum value to functional $J$. This solution is given by Theorem 3 .

Lemma 8 (Continuity of solutions). Let $u^{\epsilon}$ be a control perturbation around the optimal control $u^{*}$, that is, for all $t \in[a, b], u^{\epsilon}(t)=u^{*}(t)+\epsilon h(t)$, where $h(\cdot) \in L^{2}([a, b], \mathbb{R})$ is a variation and $\epsilon \in \mathbb{R}$. Denote by $x^{\epsilon}$ its corresponding state trajectory, solution of

$$
{ }_{C}^{A} D_{a, b}^{\alpha, \beta, \gamma} x^{\epsilon}(t)=f\left(t, x^{\epsilon}(t), u^{\epsilon}(t)\right), \quad x^{\epsilon}(a)=x_{a} .
$$

If $K<\frac{1}{(b-a)^{\alpha-1} M}$, where $K$ is the Lipschitz constant of $f$, then $x^{\epsilon}$ converges to the optimal state trajectory $x^{*}$ when $\epsilon$ tends to zero, that is, $x^{*}$ is continuous.

Proof. From Equation (9), we have

$$
\left|{ }_{C}^{A} D_{a, b}^{\alpha, \beta, \gamma} x^{\epsilon}(t)-{ }_{C}^{A} D_{a, b}^{\alpha, \beta, \gamma} x^{*}(t)\right|=\left|f\left(t, x^{\epsilon}(t), u^{\epsilon}(t)\right)-f\left(t, x^{*}(t), u^{*}(t)\right)\right| .
$$

Abbreviating $f^{\epsilon}-f^{*}=f\left(t, x^{\epsilon}(t), u^{\epsilon}(t)\right)-f\left(t, x^{*}(t), u^{*}(t)\right)$, it follows, by definition of combined operators (4), that

$$
\left|\gamma\left({ }_{C}^{A} D_{a^{+}}^{\alpha, \beta} x^{\epsilon}(t)-{ }_{C}^{A} D_{a^{+}}^{\alpha, \beta} x^{*}(t)\right)+(1-\gamma)\left({ }_{C}^{A} D_{b^{-}}^{\alpha, \beta} x^{\epsilon}(t)-{ }_{C}^{A} D_{b^{-}}^{\alpha, \beta} x^{*}(t)\right)\right|=\left|f^{\epsilon}-f^{*}\right| .
$$


Next, since $\gamma \in[0,1]$, we obtain from (10) the two separate inequalities

$$
\left|{ }_{C}^{A} D_{a^{+}}^{\alpha, \beta} x^{\epsilon}(t)-{ }_{C}^{A} D_{a^{+}}^{\alpha, \beta} x^{*}(t)\right| \leq\left|f^{\epsilon}-f^{*}\right|
$$

and

$$
\left|{ }_{C}^{A} D_{b^{-}}^{\alpha, \beta} x^{\epsilon}(t)-{ }_{C}^{A} D_{b^{-}}^{\alpha, \beta} x^{*}(t)\right| \leq\left|f^{\epsilon}-f^{*}\right| .
$$

Therefore, considering (12), we can deduce the integral relation

$$
\left|x^{\epsilon}(t)-x^{*}(t)\right| \leq{ }^{\bar{A}} I_{b^{-}}^{\alpha, \beta}\left(\left|f^{\epsilon}-f^{*}\right|\right) .
$$

By the Lipschitz property of $f$, we determine that for each $t \in[a, b]$, there exists $B_{1}, B_{2} \subset \mathbb{R}$, neighbourhood of $x^{*}(t), u^{*}(t)$, respectively, and such that

$$
\begin{aligned}
\left|x^{\epsilon}(t)-x^{*}(t)\right| & \leq{ }^{\bar{A}} I_{b^{-}}^{\alpha, \beta}\left(K\left|x^{\epsilon}(t)-x^{*}(t)\right|+K|\epsilon h(t)|\right) \\
& =K|\epsilon|^{\bar{A}} I_{b^{-}}^{\alpha, \beta}(|h(t)|)+K^{\bar{A}} I_{b^{-}}^{\alpha, \beta}\left(\left|x^{\epsilon}(t)-x^{*}(t)\right|\right) .
\end{aligned}
$$

Now, applying Gronwall's inequality (Theorem 1), with $\gamma=0$, we have

$$
\begin{aligned}
\left|x^{\epsilon}(t)-x^{*}(t)\right| & \leq K|\epsilon|^{\bar{A}} I_{b^{-}}^{\alpha, \beta}(|h(t)|)+\sum_{k=1}^{\infty} K^{k}\left[\bar{A} I_{b^{-}}^{k \alpha, \beta}\left(K|\epsilon|^{\bar{A}} I_{b^{-}}^{\alpha, \beta}(|h(t)|)\right)\right] \\
& =|\epsilon| K\left[{ }^{\bar{A}} I_{b^{-}}^{\alpha, \beta}(|h(t)|)+\sum_{k=1}^{\infty} K^{k}\left({ }^{\bar{A}} I_{b^{-}}^{(k+1) \alpha, \beta}(|h(t)|)\right)\right] .
\end{aligned}
$$

Moreover, using a similar method of reasoning, we may consider (11) and obtain

$$
\left|x^{\epsilon}(t)-x^{*}(t)\right| \leq|\epsilon| K\left[\bar{A} I_{a^{+}}^{\alpha, \beta}(|h(t)|)+\sum_{k=1}^{\infty} K^{k}\left(\bar{A} I_{a^{+}}^{(k+1) \alpha, \beta}(|h(t)|)\right)\right] .
$$

Hence, summing altogether, we get

$$
\left|x^{\epsilon}(t)-x^{*}(t)\right| \leq \frac{1}{2}|\epsilon| K Y(t),
$$

where

$$
Y(t)=\left[\left({ }^{\bar{A}} I_{a^{+}}^{\alpha, \beta}+{ }^{\bar{A}} I_{b^{-}}^{\alpha, \beta}\right)(|h(t)|)\right]+\left[\sum_{k=1}^{\infty} K^{k}\left({ }^{\bar{A}} I_{a^{+}}^{\alpha, \beta}+{ }^{\bar{A}} I_{b^{-}}^{\alpha, \beta}\right)(|h(t)|)\right], \quad t \in[a, b] .
$$

Finally, when $\epsilon \rightarrow 0$, we obtain $x^{\epsilon}(t) \rightarrow x^{*}(t)$ for all $t \in[a, b]$.

Corollary 1 (Differentiability of the perturbed trajectory). There exists a function $\eta$ defined on $[a, b]$ such that

$$
x^{\epsilon}(t)=x^{*}(t)+\epsilon \eta(t)+o(\epsilon) .
$$

Proof. Since $f \in C^{1}$, we have that

$$
\begin{aligned}
f\left(t, x^{\epsilon}, u^{\epsilon}\right)=f\left(t, x^{*}, u^{*}\right)+\left(x^{\epsilon}-x^{*}\right) \frac{\partial f\left(t, x^{*}, u^{*}\right)}{\partial x}+\left(u^{\epsilon}-u^{*}\right) & \frac{\partial f\left(t, x^{*}, u^{*}\right)}{\partial u} \\
& +o\left(\left|x^{\epsilon}-x^{*}\right|,\left|u^{\epsilon}-u^{*}\right|\right) .
\end{aligned}
$$


Observe that $u^{\epsilon}-u^{*}=\epsilon h(t), u^{\epsilon} \rightarrow u^{*}$ when $\epsilon \rightarrow 0$, and, by Lemma 8 , we have $x^{\epsilon} \rightarrow x^{*}$ when $\epsilon \rightarrow 0$. Thus, the residue term can be expressed in terms of $\epsilon$ only, that is, the residue is $o(\epsilon)$. Therefore,

$$
{ }_{C}^{A} D_{a, b}^{\alpha, \beta, \gamma} x^{\epsilon}={ }_{C}^{A} D_{a, b}^{\alpha, \beta, \gamma} x^{*}+\left(x^{\epsilon}-x^{*}\right) \frac{\partial f\left(t, x^{*}, u^{*}\right)}{\partial x}+\epsilon h(t) \frac{\partial f\left(t, x^{*}, u^{*}\right)}{\partial u}+o(\epsilon),
$$

which leads to

$$
\lim _{\epsilon \rightarrow 0}\left[\frac{{ }_{C}^{A} D_{a, b}^{\alpha, \beta, \gamma}\left(x^{\epsilon}-x^{*}\right)}{\epsilon}-\frac{\left(x^{\epsilon}-x^{*}\right)}{\epsilon} \frac{\partial f\left(t, x^{*}, u^{*}\right)}{\partial x}-h(t) \frac{\partial f\left(t, x^{*}, u^{*}\right)}{\partial u}\right]=0,
$$

that is,

$$
{ }_{C}^{A} D_{a, b}^{\alpha, \beta, \gamma}\left(\lim _{\epsilon \rightarrow 0} \frac{x^{\epsilon}-x^{*}}{\epsilon}\right)=\lim _{\epsilon \rightarrow 0} \frac{\left(x^{\epsilon}-x^{*}\right)}{\epsilon} \frac{\partial f\left(t, x^{*}, u^{*}\right)}{\partial x}+h(t) \frac{\partial f\left(t, x^{*}, u^{*}\right)}{\partial u} .
$$

Now, it remains to prove the existence of the limit $\lim _{\epsilon \rightarrow 0} \frac{x^{\epsilon}-x^{*}}{\epsilon}=: \eta$. It is easy to see that the limit $\eta$ exists, as a solution of the following fractional differential equation:

$$
\left\{\begin{array}{l}
{ }_{C}^{A} D_{a, b}^{\alpha, \beta, \gamma} \eta(t)=\frac{\partial f\left(t, x^{*}, u^{*}\right)}{\partial x} \eta(t)+\frac{\partial f\left(t, x^{*}, u^{*}\right)}{\partial u} h(t), \\
\eta(a)=0 .
\end{array}\right.
$$

This ends the proof.

The following result is a necessary optimality condition for the analytic kernel fractional optimal control problem (8).

Theorem 2 (Pontryagin Maximum Principle for (8)). If $\left(x^{*}(\cdot), u^{*}(\cdot)\right)$ is an optimal pair for (8), then there exists $\lambda \in L^{\alpha, \beta}([a, b], \mathbb{R})$, called the adjoint function variable, such that the following conditions hold in the interval $[a, b]$ :

- the optimality condition

$$
u^{*} \text { maximizes, over } \Omega(t) \text {, the function, } u \mapsto H\left(t, x^{*}(t), u, \lambda(t)\right) \text {; }
$$

- $\quad$ the adjoint equation

$$
\underset{R L}{A} D_{a, b}^{\alpha, \beta, 1-\gamma} \lambda(t)=\frac{\partial H}{\partial x}\left(t, x^{*}(t), u^{*}(t)\right) ;
$$

- the transversality condition

$$
(1-\gamma)^{\bar{A}} I_{a^{+}}^{1-\alpha, \beta} \lambda(b)+\gamma^{\bar{A}} I_{b^{-}}^{1-\alpha, \beta} \lambda(b)=0,
$$

where $\bar{A}$ is such that $A_{\Gamma}\left(x^{*}\right) \cdot \bar{A}_{\Gamma}\left(x^{*}\right)=1$ and $H(t, x, u, \lambda)=L+\lambda f$.

Proof. Let $\left(x^{*}(\cdot), u^{*}(\cdot)\right)$ be solution of problem $(8)$, and $h \in L^{2}([a, b], \mathbb{R})$ be a variation, that is,

$$
h \in V:=\left\{v \in L^{2}: u^{*}+\epsilon v \in \Omega(t), \text { for any } \epsilon>0 \text { sufficiently small }\right\} .
$$

Set $u^{\epsilon}(t)=u^{*}(t)+\epsilon h(t)$, so that $u^{\epsilon} \in \Omega(t)$ and let $x^{\epsilon}$ be the state corresponding to the control $u^{\epsilon}$, that is, the solution of

$$
{ }_{C}^{A} D_{a, b}^{\alpha, \beta, \gamma} x^{\epsilon}(t)=f\left(t, x^{\epsilon}(t), u^{\epsilon}(t)\right), \quad x^{\epsilon}(a)=x_{a} .
$$


Note that $u^{\epsilon}(t) \rightarrow u^{*}(t)$ for all $t \in[a, b]$ whenever $\epsilon \rightarrow 0$. Furthermore,

$$
\left.\frac{\partial u^{\epsilon}(t)}{\partial \epsilon}\right|_{\epsilon=0}=h(t)
$$

Something similar is also true for $x^{\epsilon}$ : this is justified by Lemma 8. Indeed, because $f \in C^{1}$ with Lipschitz constant $K$ satisfying $K<\frac{1}{(b-a)^{\alpha-1} M}$, it follows from Lemma 8 that, for each fixed $t, x^{\epsilon}(t) \rightarrow x^{*}(t)$ as $\epsilon \rightarrow 0$. Moreover, by Corollary 1, the derivative $\left.\frac{\partial x^{\epsilon}(t)}{\partial \epsilon}\right|_{\epsilon=0}$ exists for each $t$. The objective functional at $\left(x^{\epsilon}, u^{\epsilon}\right)$ is

$$
J\left(x^{\epsilon}, u^{\epsilon}\right)=\int_{a}^{b} L\left(t, x^{\epsilon}(t), u^{\epsilon}(t)\right) d t
$$

Next, we introduce the adjoint function $\lambda$. Let $\lambda(\cdot)$ be in $L^{\alpha, \beta}([a, b], \mathbb{R})$, to be determined. By the integration by parts Formula (6),

$$
\begin{array}{r}
\int_{a}^{b} \lambda(t) \cdot{ }_{C}^{A} D_{a, b}^{\alpha, \beta, \gamma} x^{\epsilon}(t) d t=\gamma\left[x^{\epsilon}(t) \cdot{ }^{\bar{A}} I_{b^{-}}^{1-\alpha, \beta} \lambda(t)\right]_{a}^{b}+(1-\gamma)\left[x^{\epsilon}(t) \cdot{ }^{\bar{A}} I_{a^{+}}^{1-\alpha, \beta} \lambda(t)\right]_{a}^{b} \\
+\int_{a}^{b} x^{\epsilon}(t) \cdot{ }_{R L}^{A} D_{a, b}^{\alpha, \beta, 1-\gamma} \lambda(t) d t
\end{array}
$$

and one has

$$
\begin{aligned}
\int_{a}^{b} \lambda(t) \cdot{ }_{C}^{A} D_{a, b}^{\alpha, \beta, \gamma} x^{\epsilon}(t) d t-\gamma\left[x^{\epsilon}(t) \cdot{ }^{\bar{A}} I_{b^{-}}^{1-\alpha, \beta} \lambda(t)\right]_{a}^{b} & -(1-\gamma)\left[x^{\epsilon}(t) \cdot{ }^{\bar{A}} I_{a^{+}}^{1-\alpha, \beta} \lambda(t)\right]_{a}^{b} \\
& -\int_{a}^{b} x^{\epsilon}(t) \cdot{ }_{R L}^{A} D_{a, b}^{\alpha, \beta, 1-\gamma} \lambda(t) d t=0 .
\end{aligned}
$$

Adding this zero to the expression of $J\left(x^{\epsilon}, u^{\epsilon}\right)$ gives

$$
\begin{aligned}
\phi(\epsilon)= & J\left(x^{\epsilon}, u^{\epsilon}\right) \\
= & \int_{a}^{b}\left[L\left(t, x^{\epsilon}(t), u^{\epsilon}(t)\right)+\lambda(t) \cdot{ }_{C}^{A} D_{a, b}^{\alpha, \beta, \gamma} x^{\epsilon}(t)-x^{\epsilon}(t) \cdot{ }_{R L}^{A} D_{a, b}^{\alpha, \beta, 1-\gamma} \lambda(t)\right] d t \\
& -x^{\epsilon}(b) \cdot\left[(1-\gamma)^{\bar{A}} I_{a^{+}}^{1-\alpha, \beta} \lambda(b)+\gamma^{\bar{A}} I_{b^{-}}^{1-\alpha, \beta} \lambda(b)\right] \\
& +x^{\epsilon}(a) \cdot\left[(1-\gamma)^{\bar{A}} I_{a^{+}}^{1-\alpha, \beta} \lambda(a)+\gamma^{\bar{A}} I_{b^{-}}^{1-\alpha, \beta} \lambda(a)\right],
\end{aligned}
$$

which by (16) is equivalent to

$$
\begin{aligned}
\phi(\epsilon)= & J\left(x^{\epsilon}, u^{\epsilon}\right) \\
= & \int_{a}^{b}\left[L\left(t, x^{\epsilon}(t), u^{\epsilon}(t)\right)+\lambda(t) \cdot f\left(t, x^{\epsilon}(t), u^{\epsilon}(t)\right)-x^{\epsilon}(t) \cdot A{ }_{R L}^{A} D_{a, b}^{\alpha, \beta, 1-\gamma} \lambda(t)\right] d t \\
& -x^{\epsilon}(b) \cdot\left[(1-\gamma)^{\bar{A}} I_{a^{+}}^{1-\alpha, \beta} \lambda(b)+\gamma^{\bar{A}} I_{b^{-}}^{1-\alpha, \beta} \lambda(b)\right] \\
& +x^{\epsilon}(a) \cdot\left[(1-\gamma)^{\bar{A}} I_{a^{+}}^{1-\alpha, \beta} \lambda(a)+\gamma^{\bar{A}} I_{b^{-}}^{1-\alpha, \beta} \lambda(a)\right] .
\end{aligned}
$$

Since the maximum of $J$ occurs at $\left(x^{*}, u^{*}\right)=\left(x^{0}, u^{0}\right)$, we have that for every feasible direction's variation (i.e., $h \in V$ ), the derivative of $\phi(\epsilon)$ with respect to $\epsilon$ at $\epsilon=0$ must be negative [25], that is, 


$$
\begin{aligned}
& 0 \geqslant \phi^{\prime}(0)=\left.\frac{d}{d \epsilon} J\left(x^{\epsilon}, u^{\epsilon}\right)\right|_{\epsilon=0}= \int_{a}^{b}\left[\left.\frac{\partial L}{\partial x} \frac{\partial x^{\epsilon}(t)}{\partial \epsilon}\right|_{\epsilon=0}+\left.\frac{\partial L}{\partial u} \frac{\partial u^{\epsilon}(t)}{\partial \epsilon}\right|_{\epsilon=0}\right. \\
&\left.+\lambda(t)\left(\left.\frac{\partial f}{\partial x} \frac{\partial x^{\epsilon}(t)}{\partial \epsilon}\right|_{\epsilon=0}+\left.\frac{\partial f}{\partial u} \frac{\partial u^{\epsilon}(t)}{\partial \epsilon}\right|_{\epsilon=0}\right)-\left.{ }_{R L}^{A} D_{a, b}^{\alpha, \beta, 1-\gamma} \lambda(t) \frac{\partial x^{\epsilon}(t)}{\partial \epsilon}\right|_{\epsilon=0}\right] d t \\
&-\left.\left[(1-\gamma)^{\bar{A}} I_{a^{+}}^{1-\alpha, \beta} \lambda(b)+\gamma^{\bar{A}} I_{b^{-}}^{1-\alpha, \beta} \lambda(b)\right] \frac{\partial x^{\epsilon}(b)}{\partial \epsilon}\right|_{\epsilon=0},
\end{aligned}
$$

where the partial derivatives of $L$ and $f$ with respect to $x$ and $u$ are evaluated at $\left(t, x^{*}(t), u^{*}(t)\right)$. Rearranging the terms and using (17), we obtain that

$$
\begin{aligned}
\int_{a}^{b}\left[\left.\left(\frac{\partial L}{\partial x}+\lambda(t) \frac{\partial f}{\partial x}-{ }_{R L}^{A} D_{a, b}^{\alpha, \beta, 1-\gamma} \lambda(t)\right) \frac{\partial x^{\epsilon}(t)}{\partial \epsilon}\right|_{\epsilon=0}+\left(\frac{\partial L}{\partial u}+\lambda(t) \frac{\partial f}{\partial u}\right) h(t)\right] d t & \\
- & {\left.\left[(1-\gamma)^{\bar{A}} I_{a^{+}}^{1-\alpha, \beta} \lambda(b)+\gamma^{\bar{A}} I_{b^{-}}^{1-\alpha, \beta} \lambda(b)\right] \frac{\partial x^{\epsilon}(b)}{\partial \epsilon}\right|_{\epsilon=0} \leqslant 0 . }
\end{aligned}
$$

Setting $H=L+\lambda f$, it follows that

$$
\begin{aligned}
\int_{a}^{b}\left[\left(\frac{\partial H}{\partial x}-{ }_{R L}^{A} D_{a, b}^{\alpha, \beta, 1-\gamma} \lambda(t)\right)\right. & \left.\left.\frac{\partial x^{\epsilon}(t)}{\partial \epsilon}\right|_{\epsilon=0}+\frac{\partial H}{\partial u} h(t)\right] d t \\
& -\left.\left[(1-\gamma)^{\bar{A}} I_{a^{+}}^{1-\alpha, \beta} \lambda(b)+\gamma^{\bar{A}} I_{b^{-}}^{1-\alpha, \beta} \lambda(b)\right] \frac{\partial x^{\epsilon}(b)}{\partial \epsilon}\right|_{\epsilon=0} \leqslant 0,
\end{aligned}
$$

where the partial derivatives of $H$ are evaluated at $\left(t, x^{*}(t), u^{*}(t), \lambda(t)\right)$. Now, choosing

$$
{ }_{R L}^{A} D_{a, b}^{\alpha, \beta, 1-\gamma} \lambda(t)=\frac{\partial H}{\partial x}, \quad \text { with }\left[(1-\gamma)^{\bar{A}} I_{a^{+}}^{1-\alpha, \beta} \lambda(b)+\gamma^{\bar{A}} I_{b^{-}}^{1-\alpha, \beta} \lambda(b)\right]=0,
$$

that is, given the adjoint Equation (14) and the transversality condition (15), it yields

$$
\int_{a}^{b} \frac{\partial H}{\partial u}\left(t, x^{*}(t), u^{*}(t), \lambda(t)\right) h(t) d t \leqslant 0 .
$$

Since this inequality holds for any feasible direction's variation $h(\cdot) \in V$, we obtain that the partial derivative $\frac{\partial H}{\partial u}\left(t, x^{*}(t), u^{*}(t), \lambda(t)\right)$ belongs to the normal cone to $\Omega(t)$ at $u^{*}$ (see, e.g., p. 45 of [26]), that is, mathematically,

$$
\frac{\partial H}{\partial u}\left(t, x^{*}(t), u^{*}(t), \lambda(t)\right) \in N_{\Omega(t)}\left(u^{*}\right),
$$

meaning that $u^{*}$ maximizes $u \mapsto H\left(t, x^{*}(t), u, \lambda(t)\right)$ over $\Omega(t)$, which is exactly the optimality condition (13). This completes the proof.

Remark 1. If $\Omega(t)=L^{2}$, then $N_{\Omega(t)}\left(u^{*}\right)=0$, and the optimality condition (13) is reduced to

$$
\frac{\partial H}{\partial u}\left(t, x^{*}(t), u^{*}(t), \lambda(t)\right)=0,
$$

which gives the particular result obtained in [19] (see also [27], in a different context).

Definition 5. The candidates to solutions of (8), obtained by the application of our Theorem 2, will be called (Pontryagin) extremals. 
Example 1. Let us consider the following optimal control problem:

$$
\begin{gathered}
J[x(\cdot), u(\cdot)]=\int_{0}^{2}-\left(x(t)-t^{3}\right)^{2}-\left(u(t)-\frac{t(t-2)}{\ln t}\right)^{2} d t \longrightarrow \max , \\
{ }_{C}^{A} D_{0,2}^{\frac{1}{2}, 3 i, \gamma} x(t)=\frac{1}{2} x(t)+\frac{1}{2} u(t), \quad t \in[0,2], \\
x(\cdot) \in A C^{\frac{1}{2}, 3 i}([0,2], \mathbb{R}), \quad u(\cdot) \in L^{2}([a, b], \mathbb{R}), \\
x(0)=0,
\end{gathered}
$$

with

$$
A(x)=\sum_{n=0}^{\infty} \frac{x^{n}}{\exp (\sqrt{2}) n !}=\exp (x-\sqrt{2}), \quad \text { for which } \quad M=\sup _{|x|<\sqrt{2}} \exp (x-\sqrt{2})=1 .
$$

Identifying (18) with (8), we have the following correspondence: $a=0 ; b=2 ; \alpha=\frac{1}{2} ; \beta=3 i$; $f(t, x, u)=\frac{1}{2} x+\frac{1}{2} u ;$ and $L(t, x, u)=-\left(x-t^{3}\right)^{2}-\left(u-\frac{t(t-2)}{\ln t}\right)^{2}$. Note that $f \in C^{1}$ is Lipschitz-continuous in both variables $x$ and $u$ with Lipschitz constant $K=\frac{1}{2}$. Also, the inequality $K<\frac{1}{(b-a)^{\alpha-1} M}$ holds, that is, $\frac{1}{2}<\sqrt{2}$ is satisfied. Thus, by Lemma 8 , for any control perturbation the corresponding state trajectory converges to the optimal state solution. Moreover, by defining the Hamiltonian function as

$$
H(t, x, u, \lambda)=-\left(x-t^{3}\right)^{2}-\left(u-\frac{t(t-2)}{\ln t}\right)^{2}+\frac{1}{2} \lambda(x+u),
$$

and applying Theorem 2, it follows:

- from the optimality condition $\frac{\partial H}{\partial u}=0$ (recall Remark 1$)$, that

$$
\lambda(t)=4\left(u(t)-\frac{t(t-2)}{\ln t}\right)
$$

- $\quad$ from the adjoint equation ${ }_{R L}^{A} D_{0,2}^{\frac{1}{2}, 3 i, 1-\gamma} \lambda(t)=\frac{\partial H}{\partial x}$, that

$$
{ }_{R L}^{A} D_{0,2}^{\frac{1}{2}, 3 i, 1-\gamma} \lambda(t)=-2\left(x(t)-t^{3}\right)+\frac{1}{2} \lambda(t)
$$

- from the transversality condition

$$
(1-\gamma)^{\bar{A}} I_{a^{+}}^{1-\alpha, \beta} \lambda(b)+\gamma^{\bar{A}} I_{b^{-}}^{1-\alpha, \beta} \lambda(b)=0
$$

that

$$
\left((1-\gamma)^{\bar{A}} I_{0^{+}}^{\frac{1}{2}, 3 i}+\gamma^{\bar{A}} I_{2^{-}}^{\frac{1}{2}, 3 i}\right) \lambda(2)=0 .
$$

In conclusion, we easily see that (20)-(22) are satisfied by the triple

$$
x(t)=t^{3}, \quad u(t)=\frac{t(t-2)}{\ln t}, \quad \text { and } \quad \lambda(t) \equiv 0,
$$

which is the Pontryagin extremal: a candidate to the solution of the given problem (18). 


\subsection{Sufficient Condition for Global Optimality}

We now prove a Mangasarian type theorem for the general analytic kernel fractional optimal control problem (8).

Theorem 3 (Sufficient global optimality condition). Consider the general analytic kernel fractional optimal control problem (8) with $\Omega(t)=L^{2}$. If $(x, u) \rightarrow L(t, x, u)$ and $(x, u) \rightarrow$ $f(t, x, u)$ are concave and $(\tilde{x}, \tilde{u}, \lambda)$ is a Pontryagin extremal with $\lambda(t) \geq 0, t \in[a, b]$, then

$$
J[\tilde{x}, \tilde{u}] \geq J[x, u]
$$

for any admissible pair $(x, u)$, that is, the pair $(\tilde{x}, \tilde{u})$ is the solution to problem (8).

Proof. Since $L$ is concave as a function of $x$ and $u$, we have by the gradient inequality (Lemma 4) that

$$
\begin{aligned}
L(t, \tilde{x}(t), \tilde{u}(t))-L(t, x(t), u(t)) \geq \frac{\partial L}{\partial x}(t, \tilde{x}(t), \tilde{u}(t)) & \cdot(\tilde{x}(t)-x(t)) \\
& +\frac{\partial L}{\partial u}(t, \tilde{x}(t), \tilde{u}(t)) \cdot(\tilde{u}(t)-u(t))
\end{aligned}
$$

for any control $u$ and its associated trajectory $x$. This gives

$$
\begin{aligned}
& J[\tilde{x}(\cdot), \tilde{u}(\cdot)]-J[x(\cdot), u(\cdot)]=\int_{a}^{b}[L(t, \tilde{x}(t), \tilde{u}(t))-L(t, x(t), u(t))] d t \\
& \geq \int_{a}^{b}\left[\frac{\partial L}{\partial x}(t, \tilde{x}(t), \tilde{u}(t)) \cdot(\tilde{x}(t)-x(t))+\frac{\partial L}{\partial u}(t, \tilde{x}(t), \tilde{u}(t)) \cdot(\tilde{u}(t)-u(t))\right] d t \\
& =\int_{a}^{b}\left[\frac{\partial \tilde{L}}{\partial x} \cdot(\tilde{x}(t)-x(t))+\frac{\partial \tilde{L}}{\partial u} \cdot(\tilde{u}(t)-u(t))\right] d t,
\end{aligned}
$$

where $\tilde{L}=L(t, \tilde{x}(t), \tilde{u}(t))$. From the adjoint Equation (14), we can write

$$
\frac{\partial L}{\partial x}(t, \tilde{x}(t), \tilde{u}(t))={ }_{R L}^{A} D_{a, b}^{\alpha, \beta, 1-\gamma} \lambda(t)-\lambda(t) \frac{\partial f}{\partial x}(t, \tilde{x}(t), \tilde{u}(t)),
$$

while from the optimality condition (13) (recall Remark 1) we have

$$
\frac{\partial L}{\partial u}(t, \tilde{x}(t), \tilde{u}(t))=-\lambda(t) \frac{\partial f}{\partial u}(t, \tilde{x}(t), \tilde{u}(t)) .
$$

It follows from (24) that

$$
\begin{aligned}
& J[\tilde{x}(\cdot), \tilde{u}(t)]-J[x(\cdot), u(\cdot)] \\
& \quad \geq \int_{a}^{b}\left[\left({ }_{R L}^{A} D_{a, b}^{\alpha, \beta, 1-\gamma} \lambda(t)-\lambda(t) \frac{\partial \tilde{f}}{\partial x}\right) \cdot(\tilde{x}(t)-x(t))-\lambda(t) \frac{\partial \tilde{f}}{\partial u} \cdot(\tilde{u}(t)-u(t))\right] d t,
\end{aligned}
$$

where $\tilde{f}=f(t, \tilde{x}(t), \tilde{u}(t))$. Next, by using the integration by parts Formula (6), we get

$$
\begin{aligned}
\int_{a}^{b} \lambda(t) & \cdot{ }_{C}^{A} D_{a, b}^{\alpha, \beta, \gamma}(\tilde{x}(t)-x(t)) d t=\gamma\left[(\tilde{x}(t)-x(t)) \cdot{ }^{\bar{A}} I_{b^{-}}^{1-\alpha, \beta} \lambda(t)\right]_{a}^{b} \\
& +(1-\gamma)\left[(\tilde{x}(t)-x(t)) \cdot{ }^{\bar{A}} I_{a^{+}}^{1-\alpha, \beta} \lambda(t)\right]_{a}^{b}+\int_{a}^{b}(\tilde{x}(t)-x(t)) \cdot{ }_{R L}^{A} D_{a, b}^{\alpha, \beta, 1-\gamma} \lambda(t) d t,
\end{aligned}
$$


meaning that

$$
\begin{aligned}
\int_{a}^{b}(\tilde{x}(t) & -x(t)) \cdot \stackrel{A}{R L}^{A} D_{a, b}^{\alpha, \beta, 1-\gamma} \lambda(t) d t=\int_{a}^{b} \lambda(t) \cdot{ }_{C}^{A} D_{a, b}^{\alpha, \beta, \gamma}(\tilde{x}(t)-x(t)) d t \\
& -\gamma\left[(\tilde{x}(t)-x(t)) \cdot \bar{A} I_{b^{-}}^{1-\alpha, \beta} \lambda(t)\right]_{a}^{b}-(1-\gamma)\left[(\tilde{x}(t)-x(t)) \cdot \bar{A} I_{a^{+}}^{1-\alpha, \beta} \lambda(t)\right]_{a}^{b} .
\end{aligned}
$$

Substituting (26) into (25), we get

$$
J[\tilde{x}(\cdot), \tilde{u}(\cdot)]-J[x(\cdot), u(\cdot)] \geq \int_{a}^{b} \lambda(t)\left[\tilde{f}-f-\frac{\partial \tilde{f}}{\partial x}(\tilde{x}(t)-x(t))-\frac{\partial \tilde{f}}{\partial u}(\tilde{u}(t)-u(t))\right] d t .
$$

Finally, taking into account that $\lambda(t) \geq 0$ and $f$ is concave in both $x$ and $u$, we conclude that $J[\tilde{x}(\cdot), \tilde{u}(\cdot)]-J[x(\cdot), u(\cdot)] \geq 0$.

Example 2. It is easily proved from Theorem 3 that the Pontryagin extremal (23),

$$
x(t)=t^{3}, \quad u(t)=\frac{t(t-2)}{\ln t}, \quad \text { and } \quad \lambda(t) \equiv 0,
$$

candidate to the solution of the optimal control problem (18), found in Example 1 from the application of Theorem 2, is indeed a solution to the problem (it is a global maximizer): in this case, the Hamiltonian defined in (19) is a concave function with respect to both variables $x$ and $u$ and, furthermore, $\lambda(t) \geq 0$ for all $t \in[a, b]$.

\section{Conclusions}

In this paper, we investigated, for the first time in the literature, optimal control problems with combined general analytic kernels. Main results provide strong necessary optimality conditions of Pontryagin type, valid in the class of absolutely continuous state trajectories and $L^{2}$ controls that may take values in any time-dependent close convex set. Other results include a new Gronwall inequality and a sufficient optimality condition for global maximizers. While our results provide non-trivial and useful analytical results, as here shown with a simple illustrative example, to address real-world applications it will be necessary to develop numerical methods that implement the obtained results. This opens several possible future directions of research and will be addressed elsewhere.

Author Contributions: The authors equally contributed to this paper, read and approved the final manuscript. Formal analysis, F.N. and D.F.M.T.; Investigation, F.N. and D.F.M.T.; Writing-original draft, F.N. and D.F.M.T.; Writing-review \& editing, F.N. and D.F.M.T. All authors have read and agreed to the published version of the manuscript.

Funding: This research was funded by the Portuguese Foundation for Science and Technology (FCT), grant number UIDB/04106/2020 (CIDMA). Ndaïrou was also supported by FCT through the PhD fellowship PD/BD/150273/2019.

Data Availability Statement: Not applicable.

Acknowledgments: The authors are grateful to two anonymous reviewers for several constructive remarks and questions.

Conflicts of Interest: The authors declare no conflict of interest. The funders had no role in the design of the study; in the collection, analyses, or interpretation of data; in the writing of the manuscript, or in the decision to publish the results.

\section{References}

1. Fernandez, A.; Özarslan, M.A.; Baleanu, D. On fractional calculus with general analytic kernels. Appl. Math. Comput. 2019, 354, 248-265. [CrossRef]

2. Binh, T.T.; Baleanu, D.; Luc, N.H.; Can, N.H. Determination of source term for the fractional Rayleigh-Stokes equation with random data. J. Inequal. Appl. 2019, 2019, 308. [CrossRef] 
3. Fernandez, A.; Ustaoğlu, C. On some analytic properties of tempered fractional calculus. J. Comput. Appl. Math. 2020, 366, 112400. [CrossRef]

4. Hadian Rasanan, A.H.; Bajalan, N.; Parand, K.; Rad, J.A. Simulation of nonlinear fractional dynamics arising in the modeling of cognitive decision making using a new fractional neural network. Math. Methods Appl. Sci. 2020, 43, 1437-1466. [CrossRef]

5. Babaei, A.; Jafari, H.; Banihashemi, S. Numerical solution of variable order fractional nonlinear quadratic integro-differential equations based on the sixth-kind Chebyshev collocation method. J. Comput. Appl. Math. 2020, 377, 112908. [CrossRef]

6. Kucche, K.D.; Kharade, J.P. Analysis of impulsive $\varphi$-Hilfer fractional differential equations. Mediterr. J. Math. 2020, 17, 163. [CrossRef]

7. Malinowska, A.B.; Torres, D.F.M. Fractional calculus of variations for a combined Caputo derivative. Fract. Calc. Appl. Anal. 2011, 14, 523-537. [CrossRef]

8. Klimek, M. Fractional sequential mechanics-Models with symmetric fractional derivative. Czechoslov. J. Phys. 2001, 51, 1348-1354. [CrossRef]

9. Malinowska, A.B.; Torres, D.F.M. Multiobjective fractional variational calculus in terms of a combined Caputo derivative. Appl. Math. Comput. 2012, 218, 5099-5111. [CrossRef]

10. Malinowska, A.B.; Torres, D.F.M. Towards a combined fractional mechanics and quantization. Fract. Calc. Appl. Anal. 2012, 15, 407-417. [CrossRef]

11. Odzijewicz, T.; Malinowska, A.B.; Torres, D.F.M. Fractional variational calculus with classical and combined Caputo derivatives. Nonlinear Anal. 2012, 75, 1507-1515. [CrossRef]

12. El-Nabulsi, R.A. Calculus of variations with hyperdifferential operators from Tabasaki-Takebe-Toda lattice arguments. Rev. R. Acad. Cienc. Exactas Fís. Nat. Ser. A Mat. RACSAM 2013, 107, 419-436. [CrossRef]

13. Feng, Y.Y.; Yang, X.J.; Liu, J.G. On overall behavior of Maxwell mechanical model by the combined Caputo fractional derivative. Chin. J. Phys. 2020, 66, 269-276. [CrossRef]

14. Tavares, D.; Almeida, R.; Torres, D.F.M. Combined fractional variational problems of variable order and some computational aspects. J. Comput. Appl. Math. 2018, 339, 374-388. [CrossRef]

15. Jiang, Y.; Zhang, Q.; Chen, A.; Wei, Z. Sensitivity Analysis of Optimal Control Problems Governed by Nonlinear Hilfer Fractional Evolution Inclusions. Appl. Math. Optim. 2021, 84, 3045-3082. [CrossRef]

16. Kumar, S.; Upadhyay, A. Optimal control problem for fractional stochastic delayed systems with noninstantaneous impulses. IMA J. Math. Control Inform. 2021, 38, 855-880. [CrossRef]

17. Khan, A.; Zarin, R.; Humphries, U.W.; Akgül, A.; Saeed, A.; Gul, T. Fractional optimal control of COVID-19 pandemic model with generalized Mittag-Leffler function. Adv. Differ. Equ. 2021, 387. [CrossRef]

18. Bandaliyev, R.A.; Mamedov, I.G.; Abdullayeva, A.B.; Safarova, K.H. Optimal control problem for a degenerate fractional differential equation. Lobachevskii J. Math. 2021, 42, 1239-1247. [CrossRef]

19. Ndaïrou, F.; Torres, D.F.M. Weak Pontryagin's Maximum Principle for Optimal Control Problems Involving a General Analytic Kernel. arXiv 2021, arXiv:2109.02136.

20. Magaril-Il'yaev, G.G.; Tikhomirov, V.M. Convex Analysis: Theory and Applications; American Mathematical Society: Providence, RI, USA, 2003; Volume 222, p. viii+183. [CrossRef]

21. Rockafellar, R.T. Convex Analysis; Princeton Mathematical Series, No. 28; Princeton University Press: Princeton, NJ, USA, 1970; p. xviii+451.

22. Kilbas, A.A.; Srivastava, H.M.; Trujillo, J.J. Theory and Applications of Fractional Differential Equations; North-Holland Mathematics Studies Series; Elsevier Science B.V.: Amsterdam, The Netherlands, 2006; Volume 204, p. xvi+523.

23. Ding, X.L.; Cao-Labora, D.; Nieto, J.J. A new generalized Gronwall inequality with a double singularity and its applications to fractional stochastic differential equations. Stoch. Anal. Appl. 2019, 37, 1042-1056. [CrossRef]

24. Liu, X.; Peterson, A.; Jia, B.; Erbe, L. A generalized $h$-fractional Gronwall's inequality and its applications for nonlinear $h$-fractional difference systems with 'maxima'. J. Differ. Equ. Appl. 2019, 25, 815-836. [CrossRef]

25. Okelo, N.B. On certain conditions for convex optimization in Hilbert spaces. Khayyam J. Math. 2019, 5, 108-112. [CrossRef]

26. Barbu, V. Mathematical Methods in Optimization of Differential Systems; Mathematics and its Applications Series; Kluwer Academic Publishers Group: Dordrecht, The Netherlands, 1994; Volume 310, p. x+259. [CrossRef]

27. Ndaïrou, F.; Torres, D.F.M. Distributed-Order Non-Local Optimal Control. Axioms 2020, 9, 124. [CrossRef] 